STUDI

FRANCESI

\section{Studi Francesi}

Rivista quadrimestrale fondata da Franco Simone

188 (LXIII | II) | 2019

Penser/peser le Moyen Âge entre XVe et XVIIe siècle:

parcours de recherche - sous la direction de Maurizio

Busca et Piero Andrea Martina

\title{
Noëlle Benhamou, Dossier Guy de Maupassant. Clinique de Passy 1892-1893
}

\section{Giulia Scuro}

\section{(2) OpenEdition \\ Journals}

\section{Edizione digitale}

URL: http://journals.openedition.org/studifrancesi/20126

DOI: $10.4000 /$ studifrancesi.20126

ISSN: 2421-5856

\section{Editore}

Rosenberg \& Sellier

\section{Edizione cartacea}

Data di pubblicazione: 1 août 2019

Paginazione: 383-384

ISSN: 0039-2944

Notizia bibliografica digitale

Giulia Scuro, «Noëlle Benhamou, Dossier Guy de Maupassant. Clinique de Passy 1892-1893», Studi Francesi [Online], 188 (LXIII | II) | 2019, online dal 01 février 2020, consultato il 25 janvier 2021. URL: http://journals.openedition.org/studifrancesi/20126 ; DOI: https://doi.org/10.4000/studifrancesi. 20126

Questo documento è stato generato automaticamente il 25 janvier 2021.

\section{(†) $\odot$

Studi Francesi è distribuita con Licenza Creative Commons Attribuzione - Non commerciale - Non opere derivate 4.0 Internazionale. 


\title{
Noëlle Benhamou, Dossier Guy de Maupassant. Clinique de Passy 1892-1893
}

\author{
Giulia Scuro
}

\section{NOTIZIA}

Noëlle Benhamou, Dossier Guy de Maupassant. Clinique de Passy 1892-1893, Connaissances et Savoirs, Saint-Denis, 2018, 338 pp.

1 All'indagine scientifica che da oltre un secolo ruota intorno agli ultimi anni della vita di Guy de Maupassant, nello specifico ai due anni che lo scrittore ha trascorso nella clinica psichiatrica di Passy prima della sua morte (1892-1893), si è aggiunto un tassello importante. A cura di Noëlle Benhamou, Maître de Conférences all'Université de Picardie Jules Verne, responsabile di un portale dedicato all'autore (www.maupassantiana.fr), è apparso il dossier che contiene le riproduzioni fotostatiche - e le relative trascrizioni - delle carte che documentano l'ingresso di Maupassant nella suddetta clinica e che si credevano ormai scomparse: Dossier Guy de Maupassant. Clinique de Passy 1892-1893 (Connaissances et Savoirs, Saint-Denis, 2018).

2 Nel volume curato da Benhamou si ritrovano 52 documenti del Dossier 1718, il numero di matricola assegnato a Maupassant quando nei primi giorni di gennaio del $1892 \mathrm{fu}$ affidato alle cure dei dottori Blanche e Charcot; si tratta di documenti di natura amministrativa, dei certificati di ammissione di Maupassant nell'istituto, degli scambi epistolari tra i medici e i familiari dello scrittore e tra gli stessi medici, della lettera inviata dal commissario di polizia per accertarsi del suo stato di salute, anche di alcuni ritagli di giornale, che Benhamou trascrive in appendice e che dimostrano la particolare attenzione del personale nella conservazione di ogni comunicazione relativa alla clinica e ai suoi degenti. La Loi des aliénés del 30 giugno 1838 imponeva alle strutture mediche la stesura di un registro di tutti i pazienti, eppure nella clinica di 
Passy era scomparsa ogni prova del passaggio di Maupassant dopo la sua morte. La loro disponibilità oggi è dovuta alla donazione di Jérôme Honnorat, professore di neurologia dell'Università di Lione ed erede di André Isidore Meuriot, direttore della clinica dopo Émile Blanche.

Una trafila simile aveva interessato i referti medici, rimasti nelle mani della famiglia Blanche fino al 1977. Attualmente, quella documentazione medica è consultabile nella sezione manoscritti della Bibliothèque Nationale de France; prima di allora, l'assenza di una rendicontazione aveva impedito la produzione di un'analisi scientifica sulla casa di cura più celebre di Parigi, o, come la definisce Laure Murat: «Poste d'observation sans rival sur les rapports entre folie et création» (Murat, La maison du docteur Blanche, Gallimard, 2013, p. 21). La clinica in questione aveva ospitato nell'Ottocento molti personaggi illustri del mondo letterario e artistico, da Alfred de Vigny ad Alexandre Dumas, a Théophile Gautier, ed è, presumibilmente, a questa notorietà che vanno ricondotte le cautele adoperate sugli archivi anche dopo la chiusura dello stabilimento. Nel momento in cui i registri e i referti medici sono venuti alla luce, uno studio incrociato dei documenti ha reso possibile conoscere il resoconto a dir poco quotidiano dei pazienti.

Benhamou dedica ampio spazio della sua introduzione alla ricostruzione degli eventi che hanno accompagnato l'internamento di Maupassant, in particolare al dibattito sviluppatosi sui giornali all'indomani della notizia; la studiosa mette a confronto le differenti versioni che ne furono date con i documenti in suo possesso, mostrando i casi in cui i fatti riportati dai giornalisti non potevano in alcun modo corrispondere al vero. La progressiva paralisi generale che causò l'offuscamento della vista e poi della ragione per Maupassant, è stata nel Novecento riconosciuta tra i sintomi della sifilide (contratta dallo scrittore quando aveva soli ventisei anni), ma all'epoca delle sue crisi furono in tantissimi ad esprimere le proprie opinioni, probabilmente suggestionati dalla possibilità che l'autore di Le Horla fosse preda di un delirio simile a quello da lui descritto nelle sue opere; Émile Zola, in un'intervista apparsa su "Le Matin" il 7 gennaio 1892, dichiarò che Maupassant era stato «la victime d'un atavisme redoutable».

Benhamou si interroga sulla legittimità di pubblicare documenti che dovrebbero essere tutelati dal giuramento di Ippocrate, seppure a distanza di più di un secolo, ma le sue perplessità sono vinte dallo spirito della ricerca scientifica e dalla volontà di riabilitare la fama dell'autore e della sua famiglia, considerata a torto indifferente nei confronti dell'internamento di Maupassant, come dimostrato dalle lettere. D'altro canto, lo scrupoloso lavoro della curatrice opera una severa distinzione tra la sfera letteraria e quella medica, che sembra anche volere rivendicare l'oggettività dei dati in suo possesso per restituire nuova prospettiva a uno dei più appassionati dibattiti sulla natura della follia umana nell'uomo e nella letteratura. 